\title{
BMJ Open Geographical disparities in emergency department presentations for acute respiratory infections and risk factors for presenting: a population-based cohort study of Western Australian children
}

\author{
Rosanne Barnes, ${ }^{1}$ Christopher C Blyth, ${ }^{1,2,3}$ Nicholas de Klerk, ${ }^{4}$ Wei Hao Lee, \\ Meredith L Borland, ${ }^{5,6}$ Peter Richmond, ${ }^{2,7}$ Faye J Lim, ${ }^{\circ}$ Parveen Fathima, ${ }^{1}$ \\ Hannah C Moore ${ }^{1}$
}

To cite: Barnes R, Blyth CC, de Klerk N, et al. Geographical disparities in emergency department presentations for acute respiratory infections and risk factors for presenting: a population-based cohort study of Western Australian children. BMJ Open 2019;9:e025360. doi:10.1136/ bmjopen-2018-025360

- Prepublication history and additional material for this paper are available online. To view these files, please visit the journal online (http://dx.doi. org/10.1136/bmjopen-2018025360).

Received 19 July 2018 Revised 6 December 2018 Accepted 25 January 2019

D) Check for updates

(C) Author(s) (or their employer(s)) 2019. Re-use permitted under CC BY-NC. No commercial re-use. See rights and permissions. Published by BMJ.

For numbered affiliations see end of article.

Correspondence to Dr Rosanne Barnes; rosanne.barnes@telethonkids. org.au

\section{ABSTRACT}

Introduction Studies examining acute respiratory infections (ARIs) in emergency department (EDs), particularly in rural and remote areas, are rare. This study aimed to examine the burden of ARIs among Aboriginal and non-Aboriginal children presenting to Western Australian (WA) EDs from 2002 to 2012.

Method Using a retrospective population-based cohort study linking ED records to birth and perinatal records, we examined presentation rates for metropolitan, rural and remote Aboriginal and non-Aboriginal children from 469589 births. We used ED diagnosis information to categorise presentations into ARI groups and calculated age-specific rates. Negative binomial regression was used to investigate association between risk factors and frequency of ARI presentation.

Results Overall, $26 \%$ of presentations were for ARIs. For Aboriginal children, the highest rates were for those aged $<12$ months in the Great Southern (1233 per 1000 childyears) and Pilbara regions (1088 per 1000 child-years). Rates for non-Aboriginal children were highest in children $<12$ months in the Southwest and Kimberley (400 and 375 per 1000 child-years, respectively). Presentation rates for ARI in children from rural and remote WA significantly increased over time in all age groups $<5$ years. Risk factors for children presenting to ED with ARI were: male, prematurity, caesarean delivery and residence in the Kimberley region and lower socio-economic areas. Conclusion One in four ED presentations in WA children are for ARIs, representing a significant out-of-hospital burden with some evidence of geographical disparity. Planned linkages with hospital discharge and laboratory detection data will aid in assessing the sensitivity and specificity of ARI diagnoses in ED.

\section{INTRODUCTION}

Globally, acute respiratory infections (ARIs) are responsible for approximately one in five deaths in children aged $<5$ years and are a

\section{Strengths and limitations of this study}

- This study demonstrates that emergency department presentation for acute respiratory infections is common in children and identifies population subgroups that use emergency services more frequently than others.

- We have conducted a state-wide in-depth investigation into the diagnostic information available from the emergency department data systems with regard to respiratory infections and provided age-specific presentation rates by condition and by geographic location, which can inform future disease control strategies.

- As emergency department location was not available the postcode of the child at birth was used to stratify data by location, which is a limitation of this study.

major cause of childhood morbidity. ${ }^{1}$ Most literature on the burden of ARI in Australian children comes from studies examining hospitalisation data, limiting knowledge to the severe end of the clinical spectrum. ${ }^{2-9}$ In Western Australia (WA), 25\% of Aboriginal children and $6.5 \%$ of non-Aboriginal children are hospitalised at least once for ARI, with infant hospitalisation rates of 276.1/1000 child-years in Aboriginal children and 44.7/1000 child-years in non-Aboriginal children. ${ }^{7}$ A higher infant hospitalisation rate for ARI of 426.7/1000 child-years in Aboriginal children was identified in the Northern Territory. ${ }^{4}$

Community-level data on the burden of ARI are important for prevention and policy development. Emergency department (ED) 
data are not widely available, but data systems exist in the USA, Canada, England and Australia with data availability increasing across jurisdictions. ${ }^{81011}$ In Australia, parent-reported data from both Melbourne and Brisbane has indicated an incidence rate of 0.56 ARIs per child-month in children $<2$ years. ${ }^{39}$ In New South Wales, Australia, the ED presentation rate for pertussis in children aged $<15$ years was 6/100 000 person years ${ }^{5}$ and $11400 \mathrm{ED}$ presentations for influenza were reported among all ages (including adults) between 2005 and 2008. ${ }^{8}$ In metropolitan WA, approximately $17 \%-45 \%$ of ED presentations in children aged $<15$ years between 2000 and 2003 were for acute upper respiratory infections ${ }^{2}$ and $53 \%$ of ED presentations in children $<17$ years old between 2001 and 2005 were for acute lower respiratory infections (ALRI). ${ }^{6}$

A further knowledge gap is the frequency of ARI in EDs in rural and remote Australia, where higher hospitalisation rates have been reported. ${ }^{7}$ ED ARI burden data are essential to inform health service planning and need to be considered when assessing the economic impact of interventions including vaccination.

The Western Australian Data Linkage System (WADLS) combines individual-level data across administrative datasets through probabilistic record linkage, matching records using a range of identifying variables (eg, patient name) and continuously updates datasets. ${ }^{12}$ It is one of few comprehensive systems worldwide and consistent with international benchmarks. ${ }^{12}{ }^{13}$ We aimed to examine diagnosis information from ED presentation records in order to describe the overall and age-specific burden of ARIs among Aboriginal and non-Aboriginal children presenting to WA EDs from 2002 to 2012 and compare presentation rates across WA regions. Furthermore, we aimed to examine the monthly distribution and temporal trends of ARI presentations across geographical regions and identify infant, maternal and sociodemographic risk factors for presenting to ED with ARI in WA.

\section{METHODS}

\section{Study population, design and setting}

We conducted a population-based retrospective cohort study of births in WA between 1996 and 2012. Of WA's total population of 2.6 million, $79 \%$ reside in the capital city Perth and $6.4 \%$ are Aboriginal or Torres Strait Islanders (herein referred to as Aboriginal). ${ }^{14}$ Over $60 \%$ of Aboriginal people reside in rural and remote regions compared with approximately $27 \%$ of non-Aboriginal people. ${ }^{15}$ The climate varies across WA from a Mediterranean climate in metropolitan WA (Perth and its surrounds) and the south of the State to dry desert climate in the central regions and tropical climate in the northern regions (online supplementary figure 1 ).

\section{Data linkage and population-based datasets}

Data were extracted from the Midwives Notification System, Birth and Death Registries and the Emergency Department Data Collection (EDDC) and probabilistically linked through the WADLS. ${ }^{12} 16$ The EDDC comprises data on ED activity from WA's public and private hospitals. ${ }^{17}$ The Midwives Notification System records information on pregnancy, labour and birth, and infant and maternal factors and is complete for over $99 \%$ of WA births. ${ }^{13}$ Our assembled linked dataset contained information on births and deaths in WA from 1996 to 2012 and ED presentations from across the State from birth up to age 17 years from 2002 to 2012 for children in the birth cohort.

\section{Coding of clinical data}

Five variables in the ED dataset were used to categorise ED presentations to identify the cause of presentation and specifically identify presentation for an ARI: (1) an International Classification of Diseases (ICD), version 10 code, which was the principal diagnosis, (2) a symptom code, (3) diagnosis at discharge text, (4) presenting complaint (symptom) text and (5) a major diagnostic category ('diseases and disorders of the respiratory system'). The ICD code was the most specific diagnosis variable; however, only one, if any, ICD code was recorded for a presentation. A hierarchy was applied in the order of variables presented above, where presentations were first classified using the principal diagnosis. Those presentations that were missing a principal diagnosis were classified using the symptom code, those missing both a principal diagnosis and symptom code were classified using the diagnosis at discharge and so on down the hierarchy. We maintained specific diseases as their own category if the group was large enough to analyse (category size ranged from 710 to 118251 presentations). Other less common conditions were grouped with similar conditions, for example, sinusitis and pharyngitis were included in other upper respiratory diseases. We also identified presentations that could be related to respiratory infections (eg, febrile convulsions) and respiratory infection symptoms (eg, wheeze/cough/crackles). Finally, some chronic conditions were specifically included to capture conditions that may have been masking other acute respiratory conditions or been inaccurately diagnosed due to similar symptoms (eg, chronic bronchitis). Our coding of the clinical diagnosis and symptom information resulted in 17 ARI categories. Online supplementary table 1 lists the categories with the numbers of associated presentations, the proportion of ED records that the category represented and the variables and codes used to populate the category. A hierarchy was applied in the order presented to make the categories mutually exclusive as text variables could potentially place records in two or more categories.

\section{Exposure variables}

We examined for potential risk factors for presenting to emergency with ARI in children aged $<5$ years. The following risk factors were identified a priori for their association with hospitalisation for ARI: sex, mode of delivery, gestational age, per cent optimal birth weight (POBW), 
number of previous pregnancies, maternal age, maternal smoking, Socio-Economic Index for Area (SEIFA), season of birth and geographical region of residence. The POBW measure was used as an appropriate measure of fetal growth as it takes into account the gestational duration, fetal gender, maternal age, maternal height and parity. ${ }^{18}$ As the location of the EDs were not available, residential postcode at birth was used to stratify data into geographical regions. The SEIFA used for this study was the Index of Relative Socioeconomic Advantage and Disadvantage (IRSAD), which indicates relative access to resources and ability to participate in society for households within the same collection district (approximately 200 dwellings) using information from the latest census. ${ }^{19}$ The IRSAD incorporates measures of disadvantage that can be offset by the included measures of advantage. The SEIFA score was measured at the time of the child's birth and grouped into quintiles.

\section{Statistical analysis}

Dates of birth and death were used to calculate age-specific person-time-at-risk denominators and ARI presentation rates per 1000 child-years and $95 \%$ CIs for Aboriginal and non-Aboriginal children presenting between 2002 and 2012 in each WA region: Metropolitan, South-West, Great Southern, Midwest-Murchison, Wheatbelt, Kimberley, Pilbara and Goldfields (online supplementary figure 1). To limit the likelihood that children presenting to ED may have moved from their geographical region at birth, we restricted analyses presented by region and risk factor analysis to children aged $<5$ years at time of presentation. Aboriginal status was identified using a validated algorithm in all available records for an individual. ${ }^{20}$ Seasonal distributions of presentations were examined by stratifying records by month of presentation. Annual presentation rates were calculated using the year of presentation and tested for linear age-specific annual trends over the study period using negative binomial regression. We also used negative binomial regression to calculate adjusted incidence rate ratios where the outcome is the frequency (number) of ARI presentations in the first 5 years of life for the infant and the exposures are maternal and sociodemographic risk factors. We explore the impact of all potential exposure factors at the same time in separate models for Aboriginal and non-Aboriginal children. To account for intragroup correlation with children presenting multiple times, we used the clustered sandwich estimator. ${ }^{21}$ Data cleaning was completed using IBM SPSS Statistics, V.24, with statistical analyses conducted in STATA V.14 and EpiBasic V.3. ${ }^{22}$

\section{Patient and public involvement}

This is a total population-based study examining patient records retrospectively. A community reference group of parents and other members of the general public were consulted prior to project commencement to ensure broad project outcomes were a priority to the community.

\section{RESULTS}

Almost all ED presentations ( $\mathrm{n}=1 \quad 607 \quad 825 ; 99.5 \%$ ) between 2002 and 2012 successfully linked to the birth cohort dataset. The remaining records were excluded due to inconsistent date of birth information or being related to children who were not born in WA. Records missing a postcode at the time of birth were also excluded ( $\mathrm{n}=1568)$ leaving $1606257 \mathrm{ED}$ records pertaining to 337201 children who presented to ED before age 17 years during the study period (figure 1). In the final categories, ICD codes identified $66.4 \%$ of ARI presentations, symptom codes identified a further $1.0 \%$, text variables $5.4 \%$ and the major diagnostic category $27.2 \%$. For analyses by geographical region, the cohort was restricted to

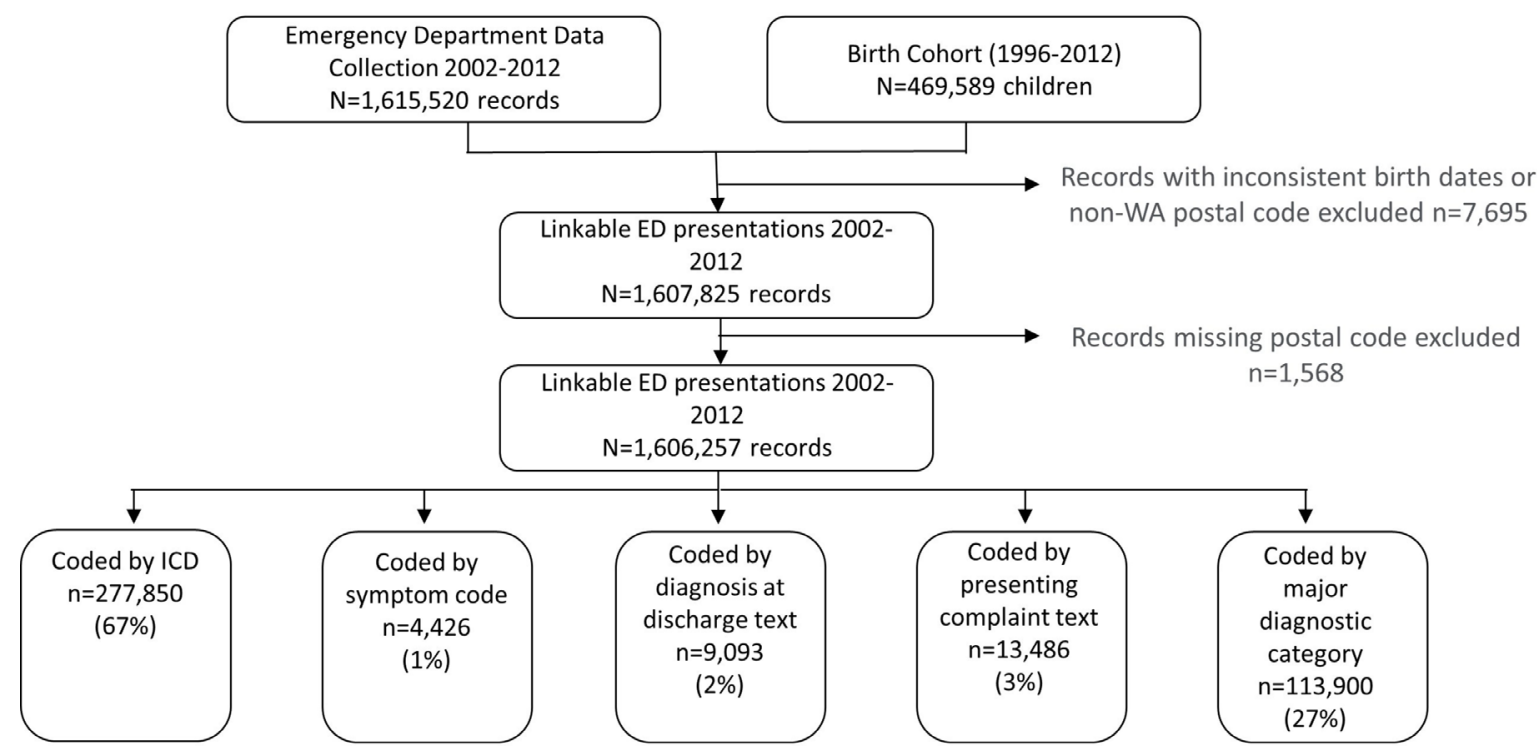

Figure 1 Selection of participants and coding of acute respiratory infections. ED, emergency department; ICD, International Classification of Diseases; WA, Western Australia. 
children presenting to ED in the first 5 years of life $(n=1$ 034924 records for 269740 children).

\section{Presentations for ARI}

Overall, 26\% of ED presentations between 2002 and 2012 in children aged $<17$ years were for ARI $(n=418755)$ and among presentations for children aged $<5$ years, $32 \%$ were for ARI $(n=332149)$. Almost three quarters $(72 \%)$ of children from the birth cohort presented to ED at least once, $40 \%$ of children presented to ED at least once for an ARI before age 17 years and $33 \%$ of children presented to ED with an ARI before their fifth birthday. There were 90421 children $<17$ years with repeat presentations for ARI (range 1-85), and in both Aboriginal and non-Aboriginal children the median number of presentations for ARI per child was one (lower quartile $=1$, upper quartile $=3$ ).

The most common diagnostic categories were respiratory disease $(\mathrm{n}=118251)$, viral illness $(\mathrm{n}=72927)$, other upper respiratory disease $(\mathrm{n}=61563)$, croup $(\mathrm{n}=32480)$ and bronchiolitis $(\mathrm{n}=22446)$. Approximately $42 \%$ of all presentations $(\mathrm{n}=670116)$ only had a major diagnostic category as diagnosis information, of which 2035 presentations were classified as 'unknown'. The major diagnostic category variable was used to classify the vast majority $(96 \%)$ of records that made up the respiratory disease category.

Table 1 presents the numbers and rates of presentations for WA children aged $<17$ years for each ARI category. Rates were higher for Aboriginal children for most ARI categories except croup and fever, where rates were higher in non-Aboriginal children. The overall presentation rate for ARI in Aboriginal children was 282/1000 child-years and in Aboriginal children $<12$ months was $1028 / 1000$ child-years. For non-Aboriginal children, the overall rate was $116 / 1000$ child-years and in those $<12$ months was 297/1000 child-years.

The respiratory disease category had the highest presentation rates for Aboriginal children in each age group, far higher than any other category (185/1000 child-years overall; table 1). The highest rates overall were observed in children $<12$ months old. For most infections or symptoms affecting the lower respiratory system including pneumonia, bronchiolitis, pertussis, unspecified ALRI, bronchitis and wheeze/cough/crackles, rates in Aboriginal children were 2-3 times higher than in non-Aboriginal children under 12 months but similar in the older age groups.

\section{Presentation for ARI across WA regions}

Diagnosis and symptom information available varied between geographical areas. In the metropolitan area, ICD coding was available for $81 \%$ of records, whereas use of these codes in EDs outside the metropolitan area ranged from $26 \%$ in the South-West to $3.4 \%$ in the Kimberley (data not shown). The South-West had the largest proportions of text variable information available (14.6\% for diagnosis at discharge text and $16.4 \%$ for presenting complaint text).
Table 2 presents the numbers and rates of total ARI presentations in each geographical region. When combined, the overall rates for non-metropolitan areas were higher than metropolitan for non-Aboriginal children (IRR for non-metropolitan to metropolitan=1.19 in children aged $<12$ months and 1.14 in children aged $1-4$ years). The highest rates in Aboriginal children were for those aged $<12$ months in the Great Southern and Pilbara regions with rates of 1233 and 1088 per 1000 child-years, respectively. For non-Aboriginal children, the highest rate was in the South-West in children aged $<12$ months (400.3/1000 child-years). The lowest rates were observed in the Goldfields in children aged 1-4 years (274.9/1000 child-years in Aboriginal children and 164.4/1000 childyears in non-Aboriginal children). In Aboriginal children, ARI rates for those $<12$ months were approximately $2-3$ times that of children 1-4 years, whereas in non-Aboriginal children rates for those $<12$ months were 1.7 times the rates for children 1-4 years.

Figure 2 shows the monthly distribution of the number of presentations for the top five categories of ARI. In the metropolitan area a clear peak of ARI presentation to ED was observed from June to September with the most number of presentations in July/August. The Midwest-Murchison with much fewer presentations showed a similar distribution peaking in July/August. Monthly presentations in the South-West, Great Southern, Wheatbelt, Goldfields and Pilbara peaked a little later in August, and the Kimberley was the only region with a bimodal distribution peaking in March and July/August. In all regions except metropolitan Perth, the majority of ED presentations were coded respiratory disease (figure 2).

Figure 3 presents the annual age-specific rates for overall ARI in Aboriginal and non-Aboriginal in metropolitan, rural and remote WA by year of presentation. There was an overall increase in ARI over time in all children from rural and remote regions. In non-Aboriginal children from the metropolitan area, increases in ARI rates were observed in those aged $1-5$ and $6-11$ months. No significant trends were observed in overall ED presentations (data not shown). Rates did fluctuate over time, particularly in Aboriginal children in the younger age groups; however, rates appear to increase in later years of the study.

Infant, maternal and sociodemographic risk factors for ARI presentation rates were similar in Aboriginal and non-Aboriginal children (table 3). The strongest risk factors associated with ARI rates in both Aboriginal and non-Aboriginal children were male sex, prematurity, caesarean delivery, birth in the Kimberley and birth in a lower socioeconomic area. Maternal age $<30$ years was also a risk factor in non-Aboriginal children and birth in the Great Southern was a risk factor in Aboriginal children. The strongest risk factor was gestational age where compared with children born $\geq 37$ weeks, the IRR for Aboriginal children born $<29$ weeks was 2.70 and for non-Aboriginal children born $<29$ weeks was 2.60 . 
Table 1 Number and rate of emergency department presentations for acute respiratory infections in Western Australian Aboriginal and non-Aboriginal children aged $<17$ years $(2002-2012)$

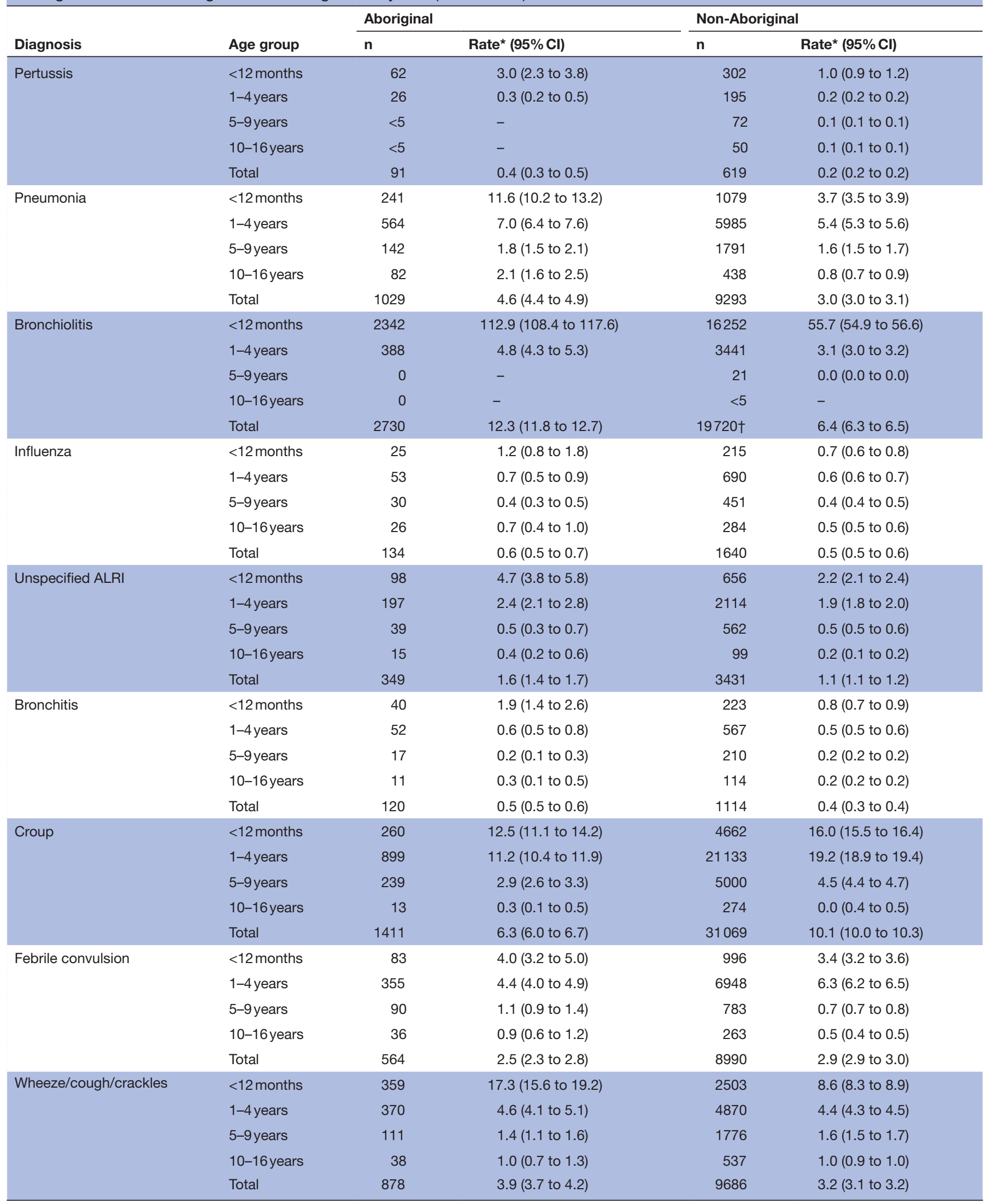


Table 1 Continued

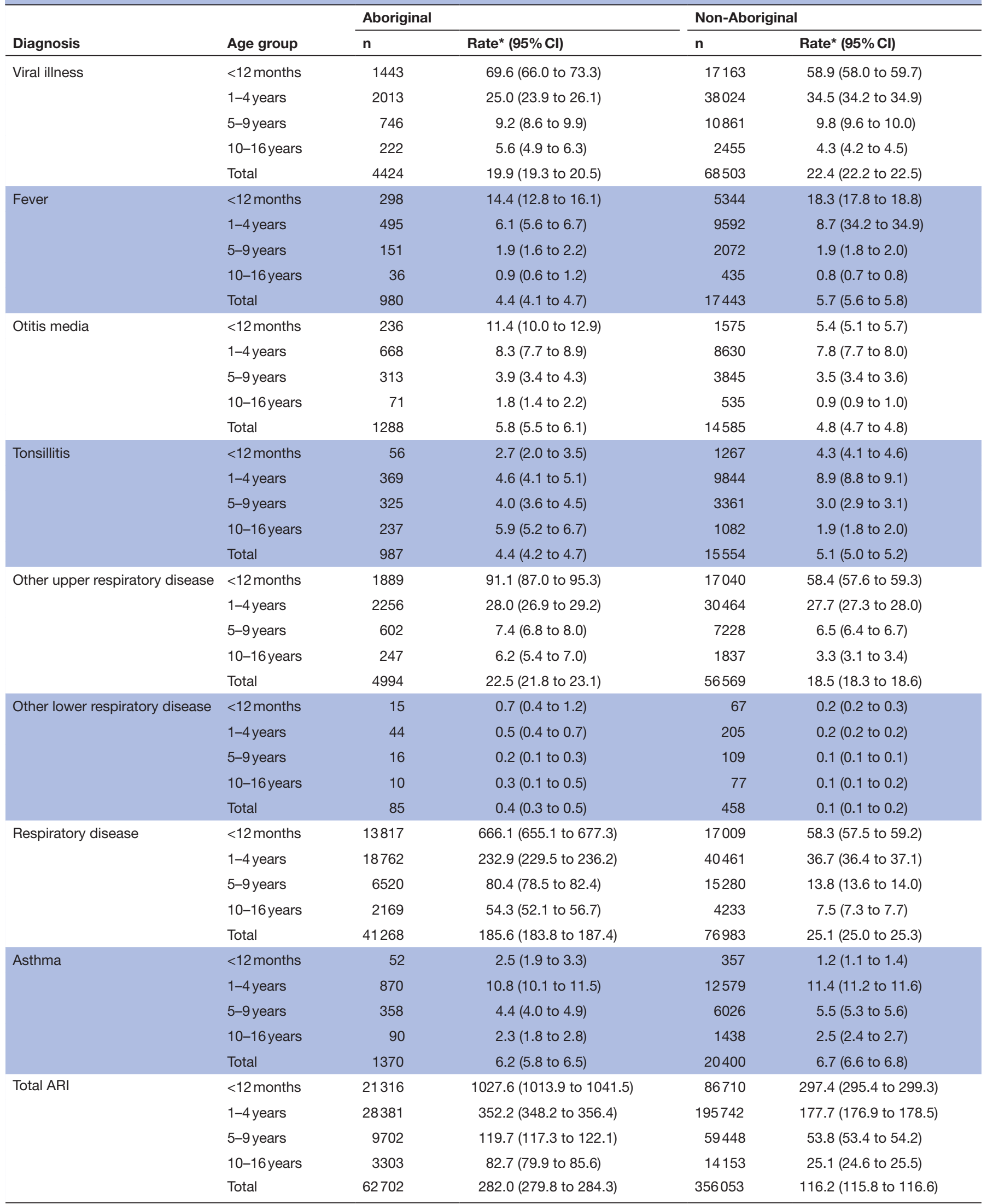

*Rate per 1000 child-years at risk from Western Australian live births.

†This total has been rounded to the nearest five to conceal small cell size numbers.

ALRI, acutelower respiratory infections; ARI, acute respiratory infections. 
Table 2 Number and rate of emergency department presentations for acute respiratory infections in Western Australian Aboriginal and non-Aboriginal children aged $<5$ years (2002-2012) by Western Australian region

\begin{tabular}{|c|c|c|c|c|c|c|}
\hline \multirow[b]{2}{*}{ Western Australian region } & \multicolumn{3}{|c|}{ Aboriginal } & \multicolumn{3}{|c|}{ Non-Aboriginal } \\
\hline & $\mathbf{N}$ & Rate* $^{*}$ & IRR (IRR 95\% CI) & $\mathbf{N}$ & Rate $^{*}$ & IRR (IRR 95\% CI) \\
\hline \multicolumn{7}{|l|}{$<12$ months } \\
\hline South-West & 729 & 934.9 & 1.03 (0.95 to 1.11$)$ & 6729 & 400.3 & 1.39 (1.35 to 1.42$)$ \\
\hline Great Southern & 752 & 1233.6 & 1.35 (1.26 to 1.46$)$ & 2345 & 314.3 & 1.09 (1.04 to 1.13$)$ \\
\hline Midwest-Murchison & 2583 & 991.3 & 1.09 (1.04 to 1.14$)$ & 2813 & 355.3 & $1.23(1.18$ to 1.28$)$ \\
\hline Goldfields & 1409 & 912.1 & 1.00 (0.95 to 1.06$)$ & 2768 & 316.0 & $1.09(1.05$ to 1.14$)$ \\
\hline Pilbara & 2136 & 1088.4 & 1.19 (1.14 to 1.25$)$ & 2007 & 324.8 & $1.12(1.07$ to 1.17$)$ \\
\hline Kimberley & 5737 & 900.0 & 0.99 (0.95 to 1.02$)$ & 1019 & 375.7 & $1.30(1.22$ to 1.38$)$ \\
\hline (Non-metropolitan) & 14375 & 965.1 & 1.06 (1.03 to 1.09$)$ & 20567 & 344.6 & $1.19(1.17$ to 1.21$)$ \\
\hline \multicolumn{7}{|l|}{$1-4$ years } \\
\hline South-West & 1071 & 356.5 & 1.14 (1.07 to 1.22$)$ & 16281 & 244.2 & 1.41 (1.38 to 1.43$)$ \\
\hline Great Southern & 1098 & 456.7 & 1.46 (1.38 to 1.56$)$ & 5540 & 184.4 & 1.06 (1.03 to 1.09$)$ \\
\hline Wheatbelt & 1375 & 352.5 & 1.13 (1.07 to 1.20$)$ & 7011 & 170.5 & 0.98 (0.96 to 1.01$)$ \\
\hline Midwest-Murchison & 3414 & 336.3 & 1.08 (1.04 to 1.12$)$ & 6365 & 197.3 & $1.14(1.11$ to 1.16$)$ \\
\hline Goldfields & 1706 & 274.9 & 0.88 (0.84 to 0.93$)$ & 5925 & 164.4 & 0.95 (0.92 to 0.97$)$ \\
\hline Pilbara & 2736 & 364.6 & 1.17 (1.12 to 1.22$)$ & 4113 & 172.8 & 1.00 (0.96 to 1.03$)$ \\
\hline Kimberley & 7896 & 322.4 & 1.03 (1.00 to 1.07$)$ & 2296 & 216.4 & 1.25 (1.20 to 1.30$)$ \\
\hline (Non-metropolitan) & 19296 & 334.7 & 1.07 (1.05 to 1.10$)$ & 47531 & 197.6 & 1.14 (1.13 to 1.15$)$ \\
\hline
\end{tabular}

${ }^{\star}$ Rates per 1000 child-years at risk from Western Australian live births. IRR, Incidence rate ratio.

\section{DISCUSSION}

Our findings suggest a substantial burden on WA EDs with approximately one in four presentations for ARI and evidence that presentation rates are increasing in children from rural and remote areas. The most common ARI presentation was respiratory disease, with bronchiolitis the most common specific diagnosis. Rates for overall ARI ED presentations were high (ranging from 25/1000 child-years in non-Aboriginal children aged 10-16 years to $1027 / 1000$ child-years in Aboriginal children aged $<12$ months). The burden in Aboriginal children was especially high, with similar disparity to non-Aboriginal children as is observed in hospitalisation rates, ${ }^{7}$ demonstrating the ongoing burden of disease in this population. There was some evidence of geographical disparity with the highest rates observed in the Northern and Southern rural regions. The monthly distributions depicted one late winter peak in numbers of ARI presentations (in July/August) in metropolitan WA and most of the rural and remote geographical regions except the Kimberley, with its tropical climate, having a bimodal distribution peaking in March and August. Risk factors for presenting to emergency with an ARI were similar in Aboriginal and non-Aboriginal children. The geographical disparity in ED presentation rates, with higher rates observed in children from most rural and remote regions in WA was consistent with findings focused on influenza among all ages in New South Wales. ${ }^{8}$ Reasons for the disparities include relative access to general practitioners and hospitals in the different regions. In particular, we have seen here increases in ED presentation rates in remote areas across all ages. It is unclear whether these increases represent a true increase in disease burden, or increases in data capture in remote areas, and there is a lack of primary healthcare data from general practices and rural and remote health clinics for comparison.

Seasonal distribution of ED presentations were consistent with patterns we have seen in metropolitan WA respiratory viral detections for respiratory syncytial virus (RSV) and influenza $A$ and $\mathrm{B}^{23}$ and hospitalisations in New South Wales for ALRI. ${ }^{24}$ The bimodal seasonal distribution of ARI presentations in the far north of WA (remote Kimberley region with a tropical climate) is similar to the distribution of RSV detections in the region and seasonal patterns observed in ARI hospitalisation in the Northern Territory of Australia. ${ }^{45}$

There are subgroups of the population that use the ED more frequently than others. While for some groups the higher risk of presentation to ED for an ARI is likely because the disease incidence is higher (eg, preterm children), for 

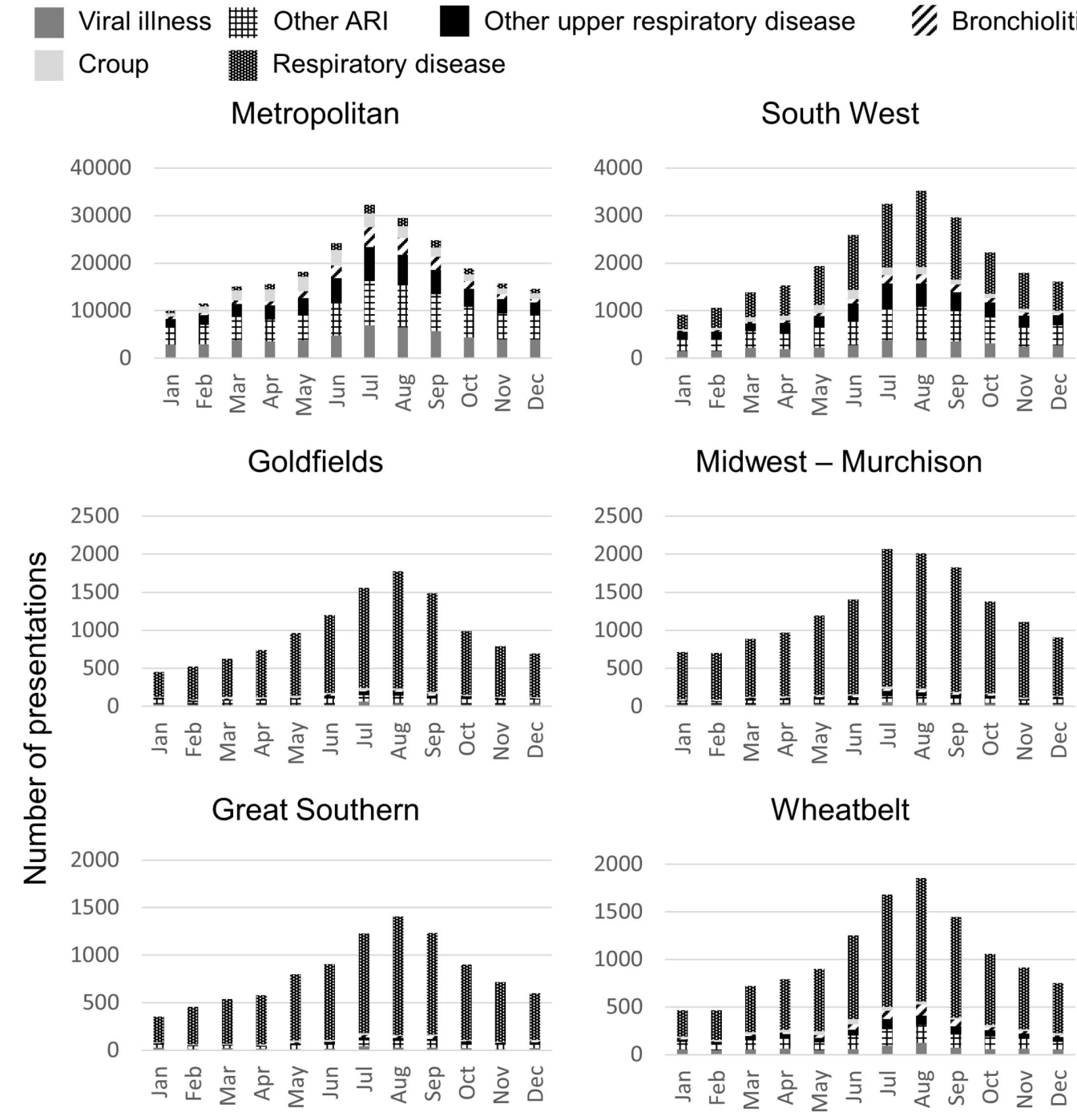

Bronchiolitis

Viral illness Other ARI Other upper respiratory disease

.

Midwest - Murchison
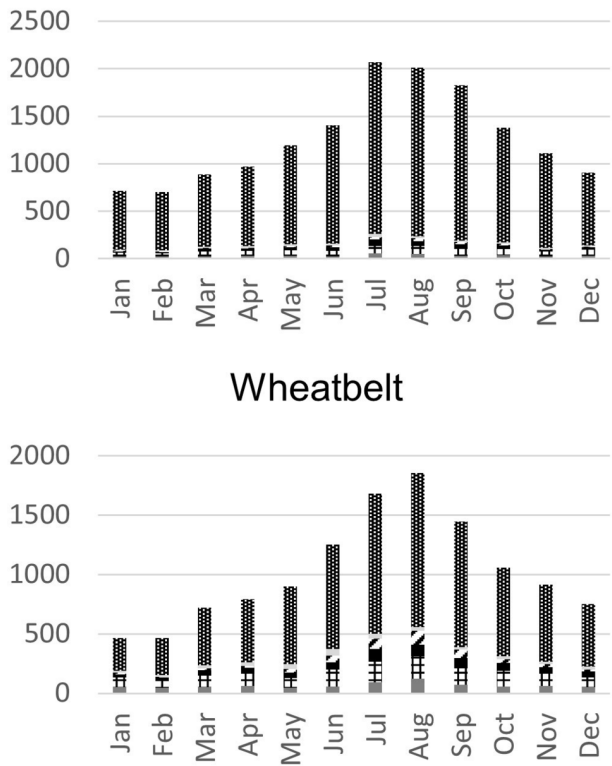

Kimberley

\section{Pilbara - Gascoyne}
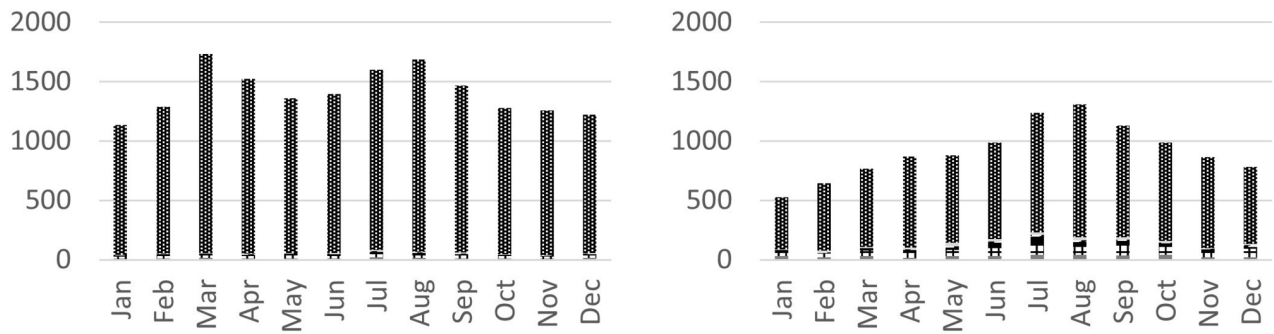

\section{Month of presentation}

Figure 2 Monthly distribution of emergency department presentations for ARIs in Aboriginal and non-Aboriginal children aged $<5$ years. ARI, acute respiratory infection.

others it could be lack of access to other primary healthcare providers, such as general practitioners in rural and remote areas. Indeed, the rate of general practitioners in rural and remote areas ranges from 53/100 000 population in the Pilbara to $141 / 100000$ population in the Kimberley compared with up to $171 / 100000$ population in Perth's inner metropolitan area. ${ }^{26}$ The risk factors for presenting with ARI to ED were similar to those previously reported risk factors for admission to hospital for ALRI including being male, being born preterm, being from a low socioeconomic area and maternal age $<20$ years. ${ }^{7}$ Two previously observed risk factors for ALRI hospitalisation, maternal smoking and higher number of previous pregnancies ${ }^{7}$ were not risk factors for presenting to ED with overall ARI. This suggests maternal smoking is associated with increased severity of disease or specifically with lower respiratory infections. Different to hospitalisation, presentation to ED may be more influenced by individual 

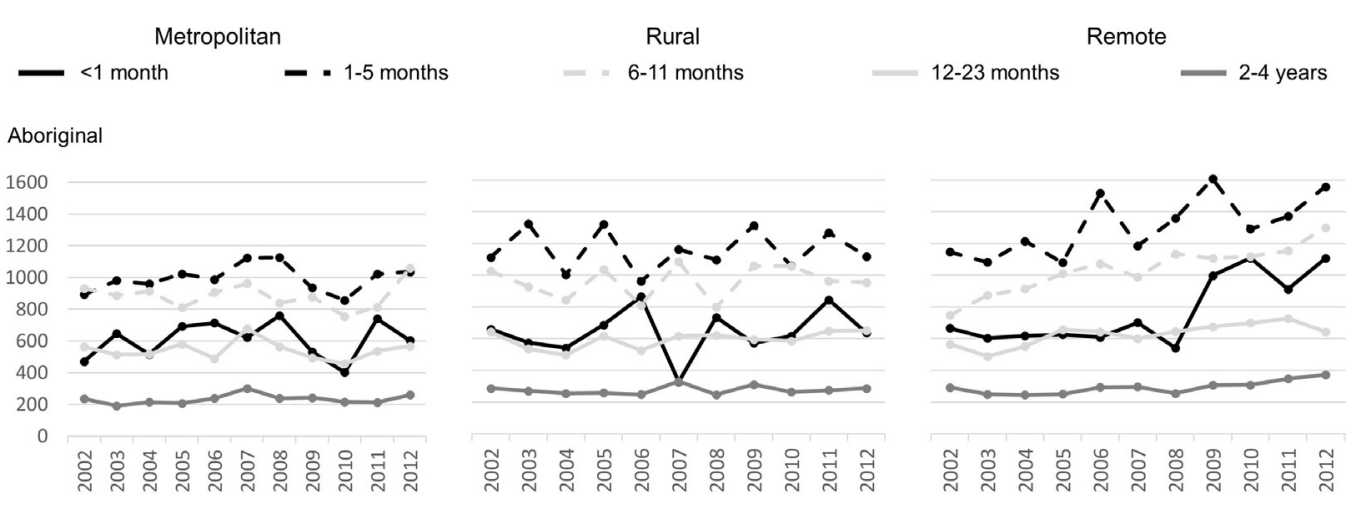

Non-Aboriginal
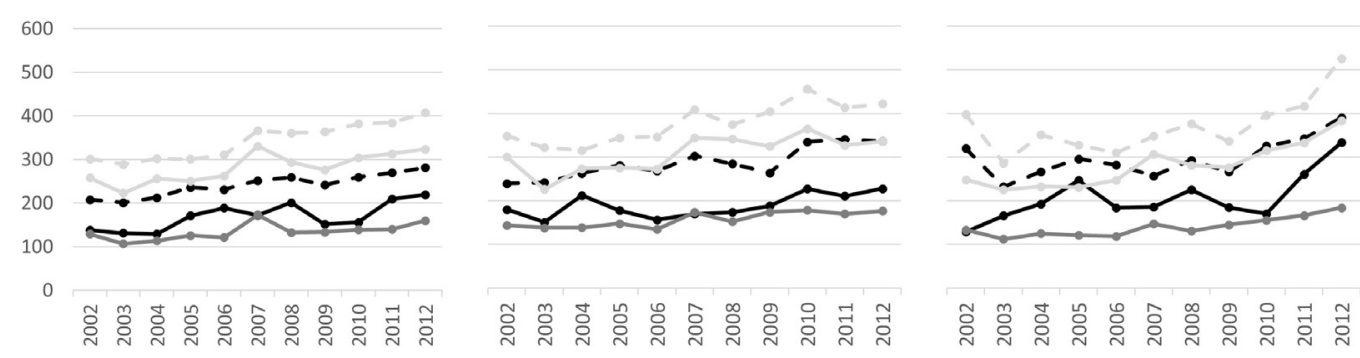

Figure 3 Annual age-specific presentation rates for acute respiratory infections in Aboriginal and non-Aboriginal children aged $<5$ years.

psychological and social factors of the child's parents or carers. Parents may be less likely to take their child to ED if they have had experiences with similar conditions in their older children and are more confident to manage their child's illness at home. Conditions presented to ED are likely to vary more in severity, whereas hospitalisations tend to be only the very severe cases and the decision to hospitalise is more likely to be made by a clinician.

Croup is a major reason for presentation to ED but has not been found to be a common diagnosis in hospitalisation. ${ }^{7}$ Rates for other specific conditions seemed low when compared with hospitalisations. This is likely a result of the high use of non-specific codes in ED, as most cases of respiratory infections are admitted with non-specific diagnoses and either discharged without testing or admitted while investigations or results are still pending. Most patients in ED will be managed based on clinical presentation, and specific diagnoses might be left to the admitting team to clarify. Rates for specific conditions are therefore likely to underestimate the burden and comparisons with hospitalisation rates for specific conditions should be made with caution. The uncertain accuracy of ICD codes in administrative datasets has previously been documented in other data linkage studies. ${ }^{5827}$ McCallum and colleagues ${ }^{5}$ noted from data linkage work that ED presentations for pertussis were likely to be missed if only coded pertussis is included due to misclassifications with other respiratory conditions and use of symptom codes for diagnosis coding. Similar results have been found when routine laboratory data are linked with hospital diagnosis data, with certain respiratory pathogens identified across a range of respiratory diagnoses ${ }^{28}$ and $38 \%$ of laboratory-confirmed hospital admissions for respiratory infections not having a respiratory infection ICD hospital diagnosis. ${ }^{27}$ The non-specific nature of the diagnostic coding also makes the severity of conditions in the ED unclear. While some presentations for severe conditions have an ICD code indicating this (eg, whooping cough), others could be just as severe but be coded as 'respiratory disease'. This is particularly likely in some remote regions that do not appear to use ICD coding. Across regions, ICD coding was available for $81 \%$ of records in metropolitan WA and in rural and remote areas ranged from $26 \%$ in the South-West to $3.4 \%$ in the Kimberley. In contrast, use of the very broad major diagnostic category in rural and remote areas ranged from $56 \%$ in the South-West to $96 \%$ in the Kimberley, versus only $12 \%$ in metropolitan WA. This highlights the inconsistencies in diagnostic practices across WA that make regional comparisons difficult and are important considerations for population-based surveillance in WA. As relatively higher proportions of Aboriginal people live in rural and remote areas compared with non-Aboriginal, rates for specific conditions may be highly underestimated in Aboriginal children. This may be why rates for some conditions, such as croup, were higher in non-Aboriginal children. Use of ICD coding in the data systems also changed over the period, with a notable increase occurring in the rural systems. Sufficiently sensitive diagnosis of ARIs in ED is likely to improve the ability to survey and manage specific conditions. The level of laboratory testing in ED is currently unknown, and linkages with laboratory data will aid in understanding the burden of specific respiratory infections. While testing results will underestimate the true burden of the specific ARIs because only a proportion of children who present are 
Open access

Table 3 Infant, maternal and sociodemographic risk factors for presenting to ED with acute respiratory infections between 2002 and 2012 among Western Australian-born Aboriginal and non-Aboriginal children $<5$ years

\begin{tabular}{|c|c|c|c|c|}
\hline \multirow[b]{2}{*}{ Risk factor } & \multicolumn{2}{|c|}{ Aboriginal } & \multicolumn{2}{|c|}{ Non-Aboriginal } \\
\hline & $\mathbf{N}$ & IRR (95\% Cl) & $\mathbf{N}$ & IRR $(95 \% \mathrm{Cl})$ \\
\hline \multicolumn{5}{|l|}{ Sex } \\
\hline Female & 22066 & Reference & 120746 & Reference \\
\hline Male & 27631 & 1.22 (1.17 to 1.28$)$ & 161706 & 1.28 (1.26 to 1.29$)$ \\
\hline \multicolumn{5}{|l|}{ Mode of delivery } \\
\hline Vaginal & 33002 & Reference & 144830 & Reference \\
\hline Instrumental & 3331 & 1.15 (1.02 to 1.06$)$ & 35055 & 1.10 (1.08 to 1.12$)$ \\
\hline Elective caesarean & 4118 & 1.20 (1.11 to 1.30$)$ & 48785 & $1.16(1.13,1.18)$ \\
\hline Emergency caesarean & 8132 & 1.17 (1.11 to 1.25$)$ & 46062 & 1.22 (1.19 to 1.24$)$ \\
\hline \multicolumn{5}{|c|}{ Per cent optimal birth weight } \\
\hline Low $(<85 \%)$ & 11697 & 1.08 (1.03 to 1.14$)$ & 31587 & 1.03 (1.01 to 1.05$)$ \\
\hline Normal $(85 \%-114 \%)$ & 29073 & Reference & 192862 & Reference \\
\hline High ( $\geq 115 \%)$ & 3441 & 1.01 (0.93 to 1.10$)$ & 25568 & 1.04 (1.02 to 1.06 ) \\
\hline \multicolumn{5}{|l|}{ Gestational age } \\
\hline$\geq 37$ weeks & 40646 & Reference & 250591 & Reference \\
\hline 33-36 weeks & 6269 & 1.13 (1.07 to 1.21$)$ & 23979 & 1.29 (1.26 to 1.33$)$ \\
\hline 29-32 weeks & 1618 & 1.61 (1.43 to 1.83$)$ & 4895 & 1.82 (1.71 to 1.94$)$ \\
\hline$<29$ weeks & 1164 & 2.70 (2.23 to 3.27$)$ & 2987 & 2.60 (2.41 to 2.82 ) \\
\hline \multicolumn{5}{|l|}{ Maternal age } \\
\hline$\geq 35$ years & 3399 & Reference & 48479 & Reference \\
\hline 30-34 years & 6337 & 0.98 (0.90 to 1.08$)$ & 79776 & 1.04 (1.02 to 1.07 ) \\
\hline 25-29years & 11399 & 1.05 (0.96 to 1.15$)$ & 82427 & 1.23 (1.21 to 1.26$)$ \\
\hline 20-24 years & 16150 & 1.09 (1.00 to 1.20$)$ & 54601 & 1.52 (1.48 to 1.56$)$ \\
\hline$<20$ years & 12412 & 1.18 (1.06 to 1.30$)$ & 17169 & 1.80 (1.73 to 1.86$)$ \\
\hline \multicolumn{5}{|l|}{$\begin{array}{l}\text { Number of previous } \\
\text { pregnancies }\end{array}$} \\
\hline 0 & 12987 & Reference & 89891 & Reference \\
\hline 1 & 10094 & 0.89 (0.83 to 0.95$)$ & 86126 & 1.01 (0.99 to 1.02 ) \\
\hline 2 & 7994 & 0.92 (0.86 to 1.00$)$ & 50911 & 1.02 (1.00 to 1.04$)$ \\
\hline$\geq 3$ & 18622 & 0.95 (0.88 to 1.02$)$ & 55524 & 1.09 (1.07 to 1.12$)$ \\
\hline \multicolumn{5}{|l|}{$\begin{array}{l}\text { Maternal smoking during } \\
\text { pregnancy }\end{array}$} \\
\hline No & 23843 & Reference & 224791 & Reference \\
\hline Yes & 24278 & 1.03 (0.99 to 1.08$)$ & 50713 & 1.14 (1.12 to 1.16$)$ \\
\hline \multicolumn{5}{|l|}{ Season of birth } \\
\hline Spring & 11157 & Reference & 68433 & Reference \\
\hline Summer & 12491 & 1.08 (1.01 to 1.14$)$ & 69096 & 1.05 (1.03 to 1.07 ) \\
\hline Autumn & 13684 & 1.10 (1.04 to 1.17$)$ & 75151 & 1.09 (1.07 to 1.11$)$ \\
\hline Winter & 12365 & 1.08 (1.02 to 1.15$)$ & 69772 & 1.03 (1.01 to 1.05 ) \\
\hline \multicolumn{5}{|l|}{ Socioeconomic index } \\
\hline $91 \%-100 \%$ & 205 & Reference & 15029 & Reference \\
\hline $76 \%-90 \%$ & 1313 & 1.09 (0.86 to 1.38$)$ & 36429 & 1.10 (1.07 to 1.14$)$ \\
\hline $26 \%-75 \%$ & 13946 & 1.21 (0.98 to 1.50$)$ & 134195 & 1.28 (1.24 to 1.31$)$ \\
\hline $11 \%-25 \%$ & 11291 & 1.30 (1.05 to 1.61$)$ & 48811 & 1.47 (1.43 to 1.52$)$ \\
\hline $0 \%-10 \%$ & 14102 & 1.19 (0.96 to 1.48$)$ & 27059 & 1.57 (1.52 to 1.63 ) \\
\hline
\end{tabular}


Table 3 Continued

\begin{tabular}{|c|c|c|c|c|}
\hline \multirow[b]{2}{*}{ Risk factor } & \multicolumn{2}{|c|}{ Aboriginal } & \multicolumn{2}{|c|}{ Non-Aboriginal } \\
\hline & $\mathbf{N}$ & IRR (95\% Cl) & $\mathbf{N}$ & IRR (95\% CI) \\
\hline \multicolumn{5}{|l|}{ Region } \\
\hline South-West & 1800 & 0.92 (0.98 to 1.21$)$ & 23010 & $1.13(1.10$ to 1.16$)$ \\
\hline Great Southern & 1850 & 1.43 (1.27 to 1.61$)$ & 7885 & $0.99(0.94$ to 1.04$)$ \\
\hline Wheatbelt & 2404 & 1.17 (1.07 to 1.29$)$ & 9897 & 0.95 (0.91 to 1.00$)$ \\
\hline Kimberley & 13633 & 1.35 (1.27 to 1.43$)$ & 3315 & $1.46(1.33$ to 1.61$)$ \\
\hline Pilbara & 4872 & 1.18 (1.09 to 1.28$)$ & 6120 & $1.11(1.05$ to 1.17$)$ \\
\hline Goldfields & 3115 & 0.92 (0.85 to 1.01$)$ & 8693 & $0.96(0.92$ to 1.00$)$ \\
\hline
\end{tabular}

All models adjusted for year of birth.

${ }^{*} 91 \%-100 \%$ represents the least disadvantaged, and 0\%-10\% represents the most disadvantaged.

ED, emergency department; IRR, Incidence rate ratio.

likely to be hospitalised or tested, it will help us to interpret the non-specific coding used in ED.

There are other limitations to our study and these data. Although the risk factor analysis was restricted to children aged less than 5 years, birth-level information, such as maternal postcode of residence at the time of her child's birth used to determine the socioeconomic index score, may not be relevant to children from older age groups. A further limitation is a lack of comorbidity information in the ED data to enable identification of children at higher risk of respiratory infections, such as those with immunocompromising conditions, chronic lung disease or neurological diseases who may experience different frequencies of ED presentations for ARI.

ED presentation for ARIs is common and has an enormous impact on the healthcare system. We have provided a comprehensive analysis of the ED burden across WA using population-based data linkage. These data from EDs across geographical areas provide essential information for ED planning, both within season and by site and to use when exploring the impact of specific interventions (eg, vaccination) or modifications to community health services (eg, establishing general practitioner after-hours clinics). Notwithstanding the limitations of clinical diagnostic accuracy, these data provide a more community-based level of the ARI burden of disease to complement previous studies assessing only hospitalisation and death at the tip of the burden of disease pyramid. There is a lack of primary healthcare data with diagnostic information in the community in general, and these ED data will be important for understanding where to target prevention strategies and form the baseline for evaluating policies.

\footnotetext{
Author affiliations

${ }^{1}$ Wesfarmers Centre of Vaccines and Infectious Diseases, Telethon Kids Institute,

The University of Western Australia, Nedlands, Australia

${ }^{2}$ Division of Paediatrics, School of Medicine, The University of Western Australia,

Perth, Western Australia, Australia

${ }^{3}$ PathWest Laboratory Medicine WA, Perth Children's Hospital, Nedlands, Australia
}

${ }^{4}$ Telethon Kids Institute, The University of Western Australia, Perth, Western Australia, Australia

${ }^{5}$ Emergency Department, Perth Children's Hospital, Nedlands, Australia

${ }^{6}$ Division of Emergency Medicine,School of Medicine, The University of Western Australia, Perth, Western Australia, Australia

${ }^{7}$ Perth Children's Hospital, Nedlands, Australia

Acknowledgements The Linkage and Client Services Team at the WA Data Linkage Branch are gratefully acknowledged for their assistance with our data acquisition, particularly Alexandra Merchant and Mikhalina Dombrovskaya as well as the data custodians of all datasets used.

Contributors RB, CCB, NdK, PR and HCM contributed to study conception, design, methods and planning. RB completed all statistical analyses, drafted the manuscript and managed revisions. CCB, WL and PR provided expert clinical advice and NdK and HCM provided statistical advice. All authors provided interpretation of the data and revisions and approved the final manuscript.

Funding This work was supported by a National Health and Medical Research Council (NHMRC) Project Grant (1045668). HCM is supported by a NHMRC Fellowship (1034254), and CCB is supported by a NHMRC Career Development Fellowship (1111596). FJL was funded by a University of Western Australia Postgraduate Award. The funding bodies were not involved in the design, conduct, analysis or reporting of this study.

Competing interests HCM reports receiving grants to their institution from the NHMRC during the conduct of this study.

Patient consent for publication Not required.

Ethics approval Ethical approval was obtained from the Western Australian Department of Health Human Research Ethics Committee and the WA Aboriginal Health Ethics Committee.

Provenance and peer review Not commissioned; externally peer reviewed. Data sharing statement No additional data available.

Open access This is an open access article distributed in accordance with the Creative Commons Attribution Non Commercial (CC BY-NC 4.0) license, which permits others to distribute, remix, adapt, build upon this work non-commercially, and license their derivative works on different terms, provided the original work is properly cited, appropriate credit is given, any changes made indicated, and the use is non-commercial. See: http://creativecommons.org/licenses/by-nc/4.0/.

\section{REFERENCES}

1. Black RE, Cousens S, Johnson HL, et al. Child Health Epidemiology Reference Group of WHO and UNICEF. Global, regional, and national causes of child mortality in 2008: a systematic analysis. Lancet 2010;375:1969-87. 
2. Ingarfield SL, Celenza A, Jacobs IG, et al. Acute upper respiratory infections in Western Australian emergency departments, 2000-2003. Aust Health Rev 2008;32:691-9.

3. Lambert SB, Allen KM, Druce JD, et al. Community epidemiology of human metapneumovirus, human coronavirus NL63, and other respiratory viruses in healthy preschool-aged children using parentcollected specimens. Pediatrics 2007;120:e929-37.

4. O'Grady KA, Torzillo PJ, Chang AB. Hospitalisation of Indigenous children in the Northern Territory for lower respiratory illness in the first year of life. Med J Aust 2010;192:586.

5. McCallum LK, Liu B, Mclntyre P, et al. Estimating the burden of pertussis in young children on hospitals and emergency departments: a study using linked routinely collected data. Epidemiol Infect 2014;142:695-705.

6. Moore HC, de Klerk N, Jacoby $\mathrm{P}$, et al. Can linked emergency department data help assess the out-of-hospital burden of acute lower respiratory infections? A population-based cohort study. BMC Public Health 2012;12: 1.

7. Moore $\mathrm{HC}$, de Klerk N, Richmond P, et al. A retrospective populationbased cohort study identifying target areas for prevention of acute lower respiratory infections in children. BMC Public Health 2010;10:757.

8. Muscatello DJ, Amin J, Maclntyre CR, et al. Inaccurate ascertainment of morbidity and mortality due to influenza in administrative databases: a population-based record linkage study. PLoS One 2014;9:e98446.

9. Sarna M, Ware RS, Sloots TP, et al. The burden of communitymanaged acute respiratory infections in the first 2-years of life. Pediatr Pulmonol 2016;51:1336-46.

10. Hall G, Krahn T, Van Dijk A, Majury A, et al. Emergency department surveillance as a proxy for the prediction of circulating respiratory viral disease in Eastern Ontario. Can J Infect Dis Med Microbiol 2013;24:150-4.

11. Harron K, Gilbert R, Cromwell D, et al. International comparison of emergency hospital use for infants: data linkage cohort study in Canada and England. BMJ Qual Saf 2018;27:31-9.

12. Holman CD, Bass AJ, Rosman DL, et al. A decade of data linkage in Western Australia: strategic design, applications and benefits of the WA data linkage system. Aust Health Rev 2008;32:766.

13. Holman CD, Bass AJ, Rouse IL, et al. Population-based linkage of health records in Western Australia: development of a health services research linked database. Aust N Z J Public Health 1999;23:453-9.
14. Australian Bureau of Statistics. Western Australia at a glance. Cat. No. 1306.5. Canberra: Australian Bureau of Statistics, 2014.

15. Australian Bureau of Statistics. Estimates of Aboriginal and Torres Strait Islander Australians, June 2016. Cat. No. 3238.0.55.001. Canberra: Australian Bureau of Statistics, 2018.

16. The Australian Version of The International Classification of Diseases, 9th Revision, Clinical Modification (ICD-9-CM). Sydney: National Coding Centre, 1996:1-4.

17. Department of Health Western Australia. Emergency Department Data Collection Data Dictionary, 2007.

18. Blair EM, Liu Y, de Klerk NH, et al. Optimal fetal growth for the Caucasian singleton and assessment of appropriateness of fetal growth: an analysis of a total population perinatal database. BMC Pediatr 2005;5:5.

19. Australian Bureau of Statistics. Socio-Economic Indexes for Areas (SEIFA). Cat. No. 2033.0.55.001. Canberra: Australian Bureau of Statistics, 2011.

20. Christensen D, Davis G, Draper G, et al. Evidence for the use of an algorithm in resolving inconsistent and missing Indigenous status in administrative data collections. Aust J Soc Issues 2014;49:423-43.

21. McCullagh P, Nelder JA. Generalized linear models. 2nd ed. London: Chapman \& Hall/CRC, 1989.

22. Juul S, Frydenberg M. EpiBasic: Aarhus University, 2011.

23. Moore HC, de Klerk N, Richmond P, et al. Seasonality of respiratory viral identification varies with age and Aboriginality in metropolitan Western Australia. Pediatr Infect Dis J 2009;28:598-603.

24. Homaira N, Oei JL, Mallitt KA, et al. High burden of RSV hospitalization in very young children: a data linkage study. Epidemiol Infect 2016;144:1612-21.

25. Hogan AB, Anderssen RS, Davis $\mathrm{S}$, et al. Time series analysis of RSV and bronchiolitis seasonality in temperate and tropical Western Australia. Epidemics 2016;16:49-55.

26. Department of Health Western Australia. General practice workforce supply and training in Western Australia. Western Australia: Department of Health Western Australia, 2018.

27. Lim FJ, Blyth CC, Fathima P, et al. Record linkage study of the pathogen-specific burden of respiratory viruses in children. Influenza Other Respir Viruses 2017;11:502-10.

28. Moore HC, de Klerk N, Keil AD, et al. Use of data linkage to investigate the aetiology of acute lower respiratory infection hospitalisations in children. J Paediatr Child Health 2012;48:520-8. 